Journal of Applied AnAlysis

Vol. 5, No. 1 (1999), pp. 35-58

\title{
CHEMICAL ATTACK IN FREE BOUNDARY DOMAINS
}

\author{
S. CLAIN \\ Received October 17, 1997 and, in revised form, January 28, 1998
}

\begin{abstract}
This paper presents a mathematical model for a chemical process used to machine cristal as glass or silica. A short physical description is presented from which we draw the mathematical model. We obtain a coupled parabolic equations system on a free boundary domain with a non-linear condition on the boundary. The existence and the uniqueness is proved in the one-dimensional case.
\end{abstract}

\section{Introduction}

In industrial application, chemical attack is employed in machining silica materials. The basic principle consists to attack a material like glass or quartz with a caustic fluid (phosphoric acid or fluoride acid). This is an usual method to build microprocessors using an etching technic. The process is also used to polish rough surface like glass micro-tips where the smoothness of the boundary is crucial. Experimental studies have been done to study the dissolution kinetic in order to predict the evolution of a silica surface in contact with the acid [7], [8], [1]. It is a typical free boundary problem where the surface of the solid cristal dissolved by the acid changes.

1991 Mathematics Subject Classification. 35R35, 35K55, 80A32.

Key words and phrases. Chemical attack, free boundary, weak solution.

ISSN 1425-6908 (c) Heldermann Verlag. 
Some authors propose mathematical models to describe the chemical process and the boundary evolution [9], [2], [6] but they all consider a mixture with an unique compound acting on the reactive surface. Furthermore, they assume the boundary to be a given function [5] or they take a simplified boundary condition [2].

We present a mathematical model of a multi-compounds chemical machining derived from the silica attack by a fluoride solution [8]. Conservative principle is used to formulate the diffusion processes. On the boundary, a reaction rate is given in function of the fluoride concentration $[8, \mathrm{p}$. 25] or $[1$, p. 541]. We give an existence and uniqueness result for a coupled parabolic equations system in a free boundary domain in the one dimensional case. We first prove using the Banach fixed point theorem the existence and the uniqueness of a solution for a small time interval. Then, we show that the solution can be extended for every time interval.

\section{The mathematical model}

A classical experiment of glass $\mathrm{SiO}_{2}$ disolution emploies a mixture of fluoride acid $\mathrm{HF}$ buffered with chloridric acid $\mathrm{HCl}$ in water $\mathrm{H}_{2} \mathrm{O}$. The chloridric acid is completly split up and gives the proton concentration $\mathrm{H}^{+}$ in the mixture to help the dissolution. The fluoride is partially split up in two ionic species $F^{-}$and $H F_{2}^{-}$. The reaction with the silica gives the unique product $\mathrm{SiF}_{6}^{2-}$ following the formula

$$
\mathrm{SiO}_{2}+4 \mathrm{HF}+2 \mathrm{~F}^{-} \longrightarrow \mathrm{SiF}_{6}^{2-}+2 \mathrm{H}_{2} \mathrm{O} .
$$

The rate dissolution depends both on the diffusion of the reactants and the products, and on the reaction on the boundary. It is usually admited that all the reaction is controlled by the dissolution rate on the boundary [8].

If one considers the etching in a macroscopic way - we mean that the dimensions are the container ones where the experiment occurs - it is a good approximation to assume the concentrations to be constant, but we get few informations about the geometric evolution of the boundary. In this work, we are interested with a small layer close to the reactive boundary such that the diffusion process can not be neglected. This layer is small $(10 \mu \mathrm{m})$ and could be linked to etching problems in semiconductor or optic technologies. The current section presents a general model describing the concentrations and the boundary evolution for the chemical attack process for a multi-dimensional geometry. In section three and the nexts, we only consider a particular configuration leading to a one dimensional formulation.

Let us consider an open bounded domain $\Omega_{0} \subset \mathbb{R}^{3}$ constituted of two subdomains $\Omega_{1}(t)$ and $\Omega_{2}(t)$ divided by the reactive boundary $S(t)$ where $t$ denotes the time, $t \in[0, T]$. The silica is represented by $\Omega_{2}(t)$ and the 
acid mixture is contained in $\Omega_{1}(t)$. We consider that the boundary of $\Omega_{1}(t)$ is the union of three subsets, $\Gamma_{N}$ (to fix a Neumann condition), $\Gamma_{D}$ (to fix a Dirichlet condition) and $S(t)$. We denote by $\mathbf{w}$ the reactive boundary velocity vector defined on $S(t)$ and by $\mathbf{n}$ the outward normal vector of $\partial \Omega_{1}(t) \cap S(t)$.

Remark 1.1. We can also consider some particular unbounded domain such as strips or cylinders.

For the sake of simplicity, we adopt the following notations

$$
\begin{array}{rlrl}
{\left[\mathrm{H}^{+}\right]} & =c_{1}, & {\left[\mathrm{OH}^{-}\right]=c_{2},} & {\left[\mathrm{H}_{2} \mathrm{O}\right]=c_{3},} \\
{[\mathrm{HF}]} & =c_{4}, \quad\left[\mathrm{~F}^{-}\right]=c_{5}, & {\left[\mathrm{HF}_{2}^{-}\right]=c_{6},} \\
{\left[\mathrm{SiF}_{6}^{2-}\right]} & =c_{7}, \quad\left[\mathrm{SiO}_{2}\right]=c_{8}, &
\end{array}
$$

where $c_{i}$ are the concentrations in moles per liter.

We assume that there is no $\mathrm{SiO}_{2}$ dissolved in the mixture (i.e. $c_{8}=0$ in $\Omega_{1}(t)$ ) and there is no acid in the glass (i.e. $c_{i}=0, i=1, \ldots, 7$ in $\Omega_{2}(t)$ ). In $\Omega_{2}(t)$, the silica concentration is a given constant $c_{8}=c_{8}^{0}$. We consider that the system is thermodynamically stable, hence we can apply the chemical equilibrium laws both in $\Omega_{1}(t)$ and on $S(t)$

$$
\left\{\begin{array}{l}
K_{1} c_{4}=c_{1} c_{5}, \\
K_{2} c_{6}=c_{5} c_{4}, \\
K_{3} c_{3}=c_{1} c_{2},
\end{array}\right.
$$

where $K_{1}, K_{2}$ and $K_{3}$ are given positive constants.

To draw the diffusion equations which governed the concentrations, we apply the conservative principle to the Atomic species. We do not apply the principle to the molecular species in order to avoid right-hand side terms in the equations. Atomic species conservation of hydrogen, oxygen, fluoride and silicon involes four equations in $\Omega_{1}(t)$ and four equations on $S(t)$. We assume the Fick law is satisfied by the different molecular species and we obtain in $\Omega_{1}(t)$ the following equations

$$
\begin{aligned}
& \partial_{t}\left(c_{1}+c_{2}+2 c_{3}+c_{4}+c_{6}\right)-D \Delta\left(c_{1}+c_{2}+2 c_{3}+c_{4}+c_{6}\right)=0, \\
& \partial_{t}\left(c_{2}+c_{3}\right)-D \Delta\left(c_{2}+c_{3}\right)=0 \\
& \partial_{t}\left(c_{4}+c_{5}+2 c_{6}+6 c_{7}\right)-D \Delta\left(c_{4}+c_{5}+2 c_{6}+6 c_{7}\right)=0 \\
& \partial_{t}\left(c_{7}\right)-D \Delta\left(c_{7}\right)=0
\end{aligned}
$$


and on the reactive boundary $S(t)$

$$
\begin{aligned}
& D \partial_{n}\left(c_{1}+c_{2}+2 c_{3}+c_{4}+c_{6}\right)+\left(c_{1}+c_{2}+2 c_{3}+c_{4}+c_{6}\right) \mathbf{w} \cdot \mathbf{n}=\mathbf{0}, \\
& D \partial_{n}\left(c_{2}+c_{3}\right)+\left(c_{2}+c_{3}\right) \mathbf{w} \cdot \mathbf{n}=\mathbf{2 c}_{\mathbf{8}}^{\mathbf{0}} \mathbf{w} \cdot \mathbf{n}, \\
& D \partial_{n}\left(c_{4}+c_{5}+2 c_{6}+6 c_{7}\right)+\left(c_{4}+c_{5}+2 c_{6}+6 c_{7}\right) \mathbf{w} \cdot \mathbf{n}=\mathbf{0}, \\
& D \partial_{n}\left(c_{7}\right)+\left(c_{7}\right) \mathbf{w} \cdot \mathbf{n}=\mathbf{c}_{\mathbf{8}}^{\mathbf{0}} \mathbf{w} \cdot \mathbf{n},
\end{aligned}
$$

where $\partial_{n}$ denotes the normal derivative. The right-hand side members caracterize the chemical reaction which occures on the boundary.

Remark 1.2. Several remarks justify the introduction of a unique diffusion coefficient $D$ for the compounds. First, the ionic compound diffusion coefficients are given for anion-cation couples - for example $\left(\mathrm{Cl}^{-}, \mathrm{Na}^{+}\right)$. The electrical interaction links the different ionic couples. In this way, when an anion moves, a cation must move to keep the electrical neutrality. Furthermore, the diffusion coefficient values of ionic couples are rather similar (about $10^{-9} \mathrm{~m}^{2} / \mathrm{s}$ ), so we can consider that all the ions behave like a unique compound. For the neutral molecules like $\mathrm{H}_{2} \mathrm{O}$ and $\mathrm{HF}$, the chemical equilibrium can justify the choice of a unique coefficient because the neutral compounds are decomposed into ionic species and reciprocally.

To complete the system, we add Neumann and Dirichlet conditions on $\Gamma=\Gamma_{N} \cup \Gamma_{D}$

$$
\begin{array}{ll}
\partial_{n} c_{i}=0 & i=1, \ldots, 7 \text { on } \Gamma_{N}, \\
c_{i}=c_{i}^{0} & i=1, \ldots, 7 \text { on } \Gamma_{D},
\end{array}
$$

where $c_{i}^{0}, i=1, \ldots, 7$ are given concentrations (usually equal to the initial concentrations).

Remark 1.3. We take a homogeneous Neumann condition to express the non-diffusion of the compounds outside of the solution. The Dirichlet condition is justified for the non-reactive interface far of the free boundary. Indeed, during the dissolution process, the concentrations vary only in a small strip close to the reactive boundary which the width $\delta$ depend on $D$ and the rate of dissolution (in our case $\delta \approx 10^{-2} \mathrm{~m}$ ). It is physically sensible to consider the concentrations constant at a distance greater than $\delta$.

Remark 1.4. We can also consider unbounded domains like strip and cylinder where the Dirichlet condition is replace by a limit. 
It remains to prescribe the reactive boundary evolution. A constitutive law is determined experimentally describing the rate of consumption in function of concentrations [8], [1]. Following the two previous authors, the rate is given by

$$
\mathbf{w} . \mathbf{n} \stackrel{\text { def }}{=} \widetilde{\mathbf{s}}\left(\mathbf{c}_{4}, \mathbf{c}_{5}, \mathbf{c}_{6}\right)=\mathbf{A c}_{4}+\mathbf{B c}_{6},
$$

where $A$ and $B$ are non-negative constants. At last, the previous equations associated with relation (1.1) give the problem $\mathcal{P}_{c}$.

Find $c_{1}, c_{2}, c_{5}, c_{7}:(0, T) \times \Omega_{1}(t) \mapsto\left(\mathbb{R}^{+}\right) \not$ such that

$$
\left\{\begin{array}{l}
\partial_{t}\left(c_{2}+\frac{c_{1} c_{2}}{K_{3}}\right)-D \Delta\left(c_{2}+\frac{c_{1} c_{2}}{K_{3}}\right)=0 \\
\partial_{t}\left(c_{1}+c_{2}+\frac{2 c_{1} c_{2}}{K_{3}}+\frac{c_{1} c_{5}}{K_{1}}+\frac{c_{1} c_{5}^{2}}{K_{1} K_{2}}\right) \\
\quad-D \Delta\left(c_{1}+c_{2}+\frac{2 c_{1} c_{2}}{K_{3}}+\frac{c_{1} c_{5}}{K_{1}}+\frac{c_{1} c_{5}^{2}}{K_{1} K_{2}}\right)=0 \\
\partial_{t}\left(c_{5}+\frac{c_{1} c_{5}}{K_{1}}+2 \frac{c_{1} c_{5}^{2}}{K_{1} K_{2}}+6 c_{7}\right)-D \Delta\left(c_{5}+\frac{c_{1} c_{5}}{K_{1}}+2 \frac{c_{1} c_{5}^{2}}{K_{1} K_{2}}+6 c_{7}\right)=0 \\
\partial_{t}\left(c_{7}\right)-D \Delta\left(c_{7}\right)=0 .
\end{array}\right.
$$

On the reactive boundary $] 0, T[\times S(t)$ we have

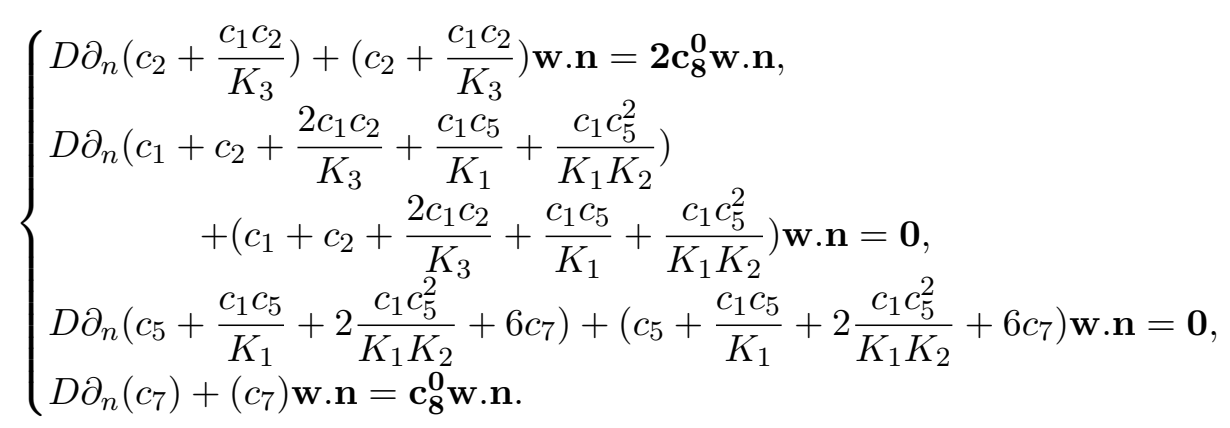

On the boundary we set the conditions

$$
\begin{array}{ll}
\partial_{n} c_{i}=0 & i=1,2,5,7 \text { on } \Gamma_{N}, \\
c_{i}=c_{i}^{0} & i=1,2,5,7 \text { on } \Gamma_{D} .
\end{array}
$$

On the reactive boundary, the rate of consumption is given by

$$
\mathbf{w} \cdot \mathbf{n}=\mathbf{s}\left(\mathbf{c}_{1}, \mathbf{c}_{2}, \mathbf{c}_{5}\right)=\mathbf{A}^{\prime} \mathbf{c}_{1} \mathbf{c}_{5}+\mathbf{B}^{\prime} \mathbf{c}_{1} \mathbf{c}_{5}^{2} .
$$

Physically, concentrations are bounded, non-negative hence we can expect that the rate of consumption is bounded, non-negative. Since $c_{7}$ does not play a part in relation (1.3), we only take $c_{1}, c_{2}, c_{5}$ into account.

In order to simplify problem $\mathcal{P}_{c}$, we introduce a new problem $\mathcal{P}_{u}$ using a non-linear transformation. We consider the mapping $F:\left(c_{1}, c_{2}, c_{5}\right) \mapsto$ 
$\left(u_{1}, u_{2}, u_{3}\right)$ defined by

$$
F(c)\left\{\begin{array}{l}
u_{1}=c_{2}+\frac{c_{1} c_{2}}{K_{3}}, \\
u_{2}=c_{1}+\frac{c_{1} c_{2}}{K_{3}}+\frac{c_{1} c_{5}}{K_{1}}+\frac{c_{1} c_{5}^{2}}{K_{1} K_{2}}, \\
u_{3}=c_{5}+\frac{c_{1} c_{5}}{K_{1}}+2 \frac{c_{1} c_{5}^{2}}{K_{1} K_{2}} .
\end{array}\right.
$$

Substracting relation $(H)$ with relation $(O)$ and relation $(F)$ with relation $6(S i)$ (resp. relation $\left(H^{\prime}\right)$ with relation $\left(O^{\prime}\right)$ and relation $\left(F^{\prime}\right)$ with relation $\left.6\left(S i^{\prime}\right)\right)$, we seek now for $u_{1}, u_{2}, u_{3}:(0, T) \times \Omega_{1}(t) \mapsto\left(\mathbb{R}^{+}\right)^{\nVdash}$ solution of the problem $\mathcal{P}_{u}$

$$
\left(\mathcal{P}_{u}\right) \begin{cases}\partial_{t} u_{i}-D \Delta u_{i}=0 & \text { in }(0, T) \times \Omega_{1}(t), \\ D \partial_{n} u_{i}=\left(a_{i}-u_{i}\right) \mathbf{w} \cdot \mathbf{n} & \text { on } S(t), \\ \partial_{n} u_{i}=0 & \text { on } \Gamma_{N}, \\ u_{i}=u_{i}^{0} & \text { on } \Gamma_{D}, \\ u_{i}=u_{i}^{0} & \text { on } \Omega_{1}(0), \\ \mathbf{w} \cdot \mathbf{n}=\mathbf{s} \circ \mathbf{F}^{-\mathbf{1}}\left(\mathbf{u}_{\mathbf{1}}, \mathbf{u}_{\mathbf{2}}, \mathbf{u}_{\mathbf{3}}\right) & \text { on } S(t),\end{cases}
$$

where $i=1,2,3$ with $a_{1}=2 c_{8}^{0}, a_{2}=-2 c_{8}^{0}, a_{3}=-6 c_{8}^{0}$ and function $s$ given by relation (1.3). We have to check that the function $r=s \circ F^{-1}$ is well-defined and to study its properties.

\section{Properties of $\mathbf{F}$}

In this section, we prove that function $F$ is a global diffeomorphism from $\left(\mathbb{R}^{+}\right)^{\nVdash}$ onto itself and $F^{-1}$ is a local Lipschitz function on $\left(\mathbb{R}^{+}\right)^{\nVdash}$ where $\mathbb{R}^{+}=[\nvdash, \infty[$.

Proposition 2.1. Let $D F$ be the jacobian matrix of $F$. For every $c_{1}, c_{2}, c_{5} \geq$ 0 , we have $|D F| \geq 1$ and $F$ is a local $C^{\infty}$ diffeomorphism from $\left(\mathbb{R}^{+}\right)^{\nVdash}$ onto $\left(\mathbb{R}^{+}\right)^{\nVdash}$.

Proof. We calculate explicitly $D F$ and obtain

$$
D F=\left(\begin{array}{ccc}
\frac{c_{2}}{K_{3}} & 1+\frac{c_{1}}{K_{3}} & 0 \\
1+\frac{c_{2}}{K_{3}}+\frac{c_{5}}{K_{1}}+\frac{c_{5}^{2}}{K_{1} K_{2}} & \frac{c_{1}}{K_{3}} & \frac{c_{1}}{K_{1}}+\frac{2 c_{1} c_{5}}{K_{1} K_{2}} \\
\frac{c_{5}}{K_{1}}+\frac{2 c_{5}^{2}}{K_{1} K_{2}} & 0 & 1+\frac{c_{1}}{K_{1}}+\frac{4 c_{1} c_{5}}{K_{1} K_{2}}
\end{array}\right) .
$$


The jacobian is given by

$$
\begin{aligned}
|D F| & =\left(1+\frac{c_{1}}{K_{3}}\right)\left[1+\frac{c_{1}}{K_{1}}+4 \frac{c_{1} c_{5}}{K_{1} K_{2}}+\frac{c_{2}}{K_{3}}+\frac{c_{1} c_{2}}{K_{1} K_{3}}+\frac{4 c_{1} c_{2} c_{5}}{K_{1} K_{2} K_{3}}+\frac{c_{5}}{K_{1}}\right. \\
& \left.+\frac{c_{5}^{2}}{K_{1} K_{2}}+\frac{c_{1} c_{5}^{2}}{K_{1}^{2} K_{2}}\right]-\frac{c_{1} c_{2}}{K_{3}}-\frac{c_{1}^{2} c_{2}}{K_{1} K_{3}^{2}}-\frac{4 c_{1}^{2} c_{2} c_{5}}{K_{1} K_{2} K_{3}^{2}} .
\end{aligned}
$$

Since $c_{1}, c_{2}$ and $c_{5}$ are non-negative, we see that $F$ is a $C^{\infty}$ function and $|D F| \geq 1$, hence $F$ is a local $C^{\infty}$ diffeomorphism from $\left(\mathbb{R}^{+}\right)^{\nVdash}$ into $\left(\mathbb{R}^{+}\right)^{\nVdash}$.

To prove $\operatorname{Im}(F)=\left(\mathbb{R}^{+}\right)^{\nVdash}$, we fix a point $\left(u_{1}, u_{2}, u_{3}\right) \in\left(\mathbb{R}^{+}\right)^{\nVdash}$ and show the existence of $\left(c_{1,0}, c_{2,0}, c_{5,0}\right)$ such that $F\left(c_{1,0}, c_{2,0}, c_{5,0}\right)=\left(u_{1}, u_{2}, u_{3}\right)$. We first define the continuous functions $c_{2}\left(c_{1}\right)$ and $c_{5}\left(c_{1}\right)$ by the unique nonnegative solutions of

$$
c_{2}-\frac{u_{1}}{1+\frac{c_{1}}{K_{3}}}=0, \quad\left(\frac{2 c_{1}}{K_{1} K_{2}}\right) c_{5}^{2}+\left(\frac{c_{1}}{K_{1}}+1\right) c_{5}-u_{3}=0
$$

and we consider the continuous function

$$
h\left(c_{1}\right) \stackrel{\text { def }}{=} c_{1}+\frac{c_{1} c_{2}\left(c_{1}\right)}{K_{3}}+\frac{c_{1} c_{5}\left(c_{1}\right)}{K_{1}}+\frac{c_{1} c_{5}^{2}\left(c_{1}\right)}{K_{1} K_{2}} .
$$

Obviously, we have $h(0)=0$ and $h\left(c_{1}\right) \rightarrow+\infty$ when $c_{1} \rightarrow \infty$. Therefore, the continuity of $h$ yields the existence of a solution $c_{1,0}$ such that $h\left(c_{1,0}\right)=u_{2}$ and relation (2.1) gives $c_{2,0}$ and $c_{5,0}$.

Proposition 2.2. The application $F$ is a global $C^{\infty}$ diffeomorphism from $\left(\mathbb{R}^{+}\right)^{\nVdash}$ onto $\left(\mathbb{R}^{+}\right)^{\nVdash}$.

Proof. We have to prove that $F$ is a one-to-one application [4, Theorem 23, p. 278]. For the sake of simplicity, we just give here the main arguments. Let us consider two distinct points $\left(c_{1}, c_{2}, c_{5}\right)$ and $\left(\widehat{c}_{1}, \widehat{c}_{2}, \widehat{c}_{5}\right)$ such that $F\left(c_{1}, c_{2}, c_{5}\right)=F\left(\widehat{c}_{1}, \widehat{c}_{2}, \widehat{c}_{5}\right)$, the first step consists to check that we must have at the same time $c_{1} \neq \widehat{c}_{1}, c_{2} \neq \widehat{c}_{2}, c_{5} \neq \widehat{c}_{5}$. Assume now for example that $c_{2}>\widehat{c}_{2}$, then from equality

$$
c_{2}+\frac{c_{1} c_{2}}{K_{3}}=\widehat{c}_{2}+\frac{\widehat{c}_{1} \widehat{c}_{2}}{K_{3}}
$$

we draw $c_{1}<\widehat{c}_{1}$. On the other hand, relation

$$
c_{5}+\frac{c_{1} c_{5}}{K_{1}}+2 \frac{c_{1} c_{5}^{2}}{K_{1} K_{2}}=\widehat{c}_{5}+\frac{\widehat{c}_{1} \widehat{c}_{5}}{K_{1}}+2 \frac{\widehat{c}_{1} \widehat{c}_{5}^{2}}{K_{1} K_{2}}
$$

and $c_{1}<\widehat{c}_{1}$ implies $c_{5}>\widehat{c}_{5}$. Using a linear combinaison of relations (2.2), (2.3) and relation

$$
c_{1}+\frac{c_{1} c_{2}}{K_{3}}+\frac{c_{1} c_{5}}{K_{1}}+\frac{c_{1} c_{5}^{2}}{K_{1} K_{2}}=\widehat{c}_{1}+\frac{\widehat{c}_{1} \widehat{c}_{2}}{K_{3}}+\frac{\widehat{c}_{1} \widehat{c}_{5}}{K_{1}}+\frac{\widehat{c}_{1} \widehat{c}_{5}^{2}}{K_{1} K_{2}}
$$


we draw

$$
c_{1}-c_{2}-c_{5}-\frac{c_{1} c_{5}^{2}}{K_{1} K_{2}}=\widehat{c}_{1}-\widehat{c}_{2}-\widehat{c}_{5}-\frac{\widehat{c}_{1} \widehat{c}_{5}^{2}}{K_{1} K_{2}} .
$$

The last equality and relation (2.3) yield

$$
\text { (i) } c_{1} c_{5}^{2}<\widehat{c}_{1} \widehat{c}_{5}^{2}, \quad \text { (ii) } c_{1} c_{5}<\widehat{c}_{1} \widehat{c}_{5}, \quad \text { (iii) } \frac{c_{1} c_{2}}{K_{3}}<\frac{\widehat{c}_{1} \widehat{c}_{2}}{K_{3}}
$$

To conclude, one can remark that equality (2.4) and relations (i), (ii), (iii) imply $c_{1}>\widetilde{c}_{1}$ and give a contradiction.

Let $s\left(c_{1}, c_{2}, c_{5}\right)$ be given by relation $(1.3)$ on $\left(\mathbb{R}^{+}\right)^{\nVdash}$, we define the rate of consumption in variables $u_{1}, u_{2}, u_{3}$ by

$$
r\left(u_{1}, u_{2}, u_{3}\right) \stackrel{\text { def }}{=} s \circ F^{-1}\left(u_{1}, u_{2}, u_{3}\right) .
$$

Notice that we have $r\left(u_{1}, u_{2}, 0\right)=0$ and $r\left(u_{1}, 0, u_{3}\right)=0$ and the function $r$ is a well-defined local Lipschitz non-negative function on $\left(\mathbb{R}^{+}\right)^{\nVdash}$. Indeed, $D F^{-1}\left(u_{1}, u_{2}, u_{3}\right)$ is bounded when $u_{1}, u_{2}$ and $u_{3}$ are bounded, and we have for every constant $M$ with $0 \leq u_{1}, u_{2}, u_{3} \leq M$ the inequalities

$$
\begin{aligned}
& r\left(u_{1}, u_{2}, u_{3}\right) \leq L u_{2}, \\
& r\left(u_{1}, u_{2}, u_{3}\right) \leq L u_{3},
\end{aligned}
$$

where $L=L(M)$ is a positive constant.

Remark 2.1. As we shall show, local existence in time of a solution just requires the function $r$ to be non-negative. On the other hand, properties (2.5) and (2.6) is fundamental to allow extention of the solution for large time. The relation means that the rate of consumption decreases when the reactant concentrations decrease. The crucial point is that the indices $i$ for which constants $a_{i}$ are negative satisfy the relation $u_{i}=0 \Rightarrow r=0$.

\section{The one dimensional problem}

In the euclidian space $O x_{1} x_{2} x_{3}$, we consider an infinite length circular cylinder of axis $O x_{1}$ orthogonal to the interface contained in the plane $\mathrm{Ox}_{2} x_{3}$. We assume that a homogeneous Neuman condition holds on the circular cylinder boundary $x_{2}^{2}+x_{3}^{2}=R^{2}$ (i. e. the compounds do not migrate outside) and we set a constant Dirichlet condition for $x_{1} \rightarrow \infty$ (i. e. the concentrations remain constant far from the reactive boundary). At $t=0$, we assume that the initial concentrations are invariant in all the $O x_{1}$-axis orthogonal planes therefore, in relation to the variables $x_{2}$ and $x_{3}$, the concentrations remain invariant during the dissolution process. 
We then replace the three dimensional problem by a one dimensional model with domain $\left.\Omega_{1}(t)=\right] \alpha(t), \infty$ [ where the interface position is described by a real function $\alpha(t)$ with $\alpha^{\prime}(t)=-r$. Let $y=x-\alpha(t)$ and set $u_{i}(t, x)=v_{i}(t, x-\alpha(t))$, we obtain a new system $\mathcal{P}_{v}$ : find $v_{1}, v_{2}, v_{3}$ : ] $0, T[\times] 0, \infty\left[\mapsto\left(\mathbb{R}^{+}\right)^{\nVdash}\right.$ such that for $i=1,2,3$

$$
\mathcal{P}_{v}\left\{\begin{array}{lc}
\partial_{t} v_{i}-\alpha^{\prime} \partial_{y} v_{i}-D \partial_{y y} v_{i}=0 & \text { in }] 0, T[\times] 0, \infty[, \\
D \partial_{n} v_{i}=\left(v_{i}-a_{i}\right) \alpha^{\prime} & \text { on } y=0, \\
\lim _{y \rightarrow \infty} v_{i}(t, y)=b_{i} \stackrel{\text { def }}{=} \lim _{y \rightarrow \infty} u_{i}^{0}(y), & \forall t \in] 0, T[, \\
v_{i}(0, y)=u_{i}^{0}(y) & \text { on }] 0, \infty[, \\
\alpha^{\prime}(t)=-r\left(v_{1}(t, 0), v_{2}(t, 0), v_{3}(t, 0)\right), & \forall t \in] 0, T[, \\
\alpha(0)=0 . &
\end{array}\right.
$$

The functions $u_{i}^{0}$ and the real numbers $b_{i}$ are non-negative. We draw the constants $a_{i}$ from the chemical reaction formula on the boundary, $a_{1}=2 c_{8}^{0}$, $a_{2}=-2 c_{8}^{0}, a_{3}=-6 c_{8}^{0}$ where $c_{8}^{0}$ is a positive real number.

Two different methods can be used to solve problem $\mathcal{P}_{v}$. The first one consists to work with Hölder spaces and the strong maximum principle. Nevertheless, we adopt here a method only based on the energy equality to obtain a solution in a weak sense. As $u_{i}^{0}(y)$ tends to a non-negative real number $b_{i}$ when $y$ tends to infinity, we perform a translation of vector $-b_{i}$ to obtain a homogeneous Dirichlet condition at infinity. We set the notations

$$
w_{i}=b_{i}-v_{i}, \quad w_{i}^{0}=b_{i}-v_{i}^{0}, \quad \nu_{i}=\max _{y \in] 0, \infty[} w_{i}^{0}(x) .
$$

Let $\phi$ be a regular real function defined on $\mathbb{R}^{+}$, we seek for functions $w_{1}, w_{2}, w_{3}$ with $w_{i} \leq b_{i}, i=1,2,3$ solutions of problem $\mathcal{P}_{w}$ 


$$
\mathcal{P}_{w}\left\{\begin{array}{lc}
\int_{0}^{\infty} \partial_{t} w_{i} \phi d y-\int_{0}^{\infty} \alpha^{\prime} \partial_{y} w_{i} \phi d y+D \int_{0}^{\infty} \partial_{y} w_{i} \partial_{y} \phi & \\
\lim _{y \rightarrow \infty} w_{i}(t, y)=0, & \\
\left.w_{i}(0, \cdot)=a_{i}-b_{i}+w_{i}(t, 0)\right] \phi(0) & \text { on }] 0, \infty[, \\
\alpha^{\prime}(t)=-r\left(v_{1}(t, 0), v_{2}(t, 0), v_{3}(t, 0)\right), & t \in] 0, T[, \\
\alpha(0)=0, &
\end{array}\right.
$$

for all $\phi$ regular enough and $i=1,2,3$. We assume that the initial molecular concentrations are positive, so we satisfy the conditions $\nu_{i}<b_{i}, i=1,2,3$.

Remark 3.1. The problem $\mathcal{P}_{\sqsupseteq}$ seems a priori rather easy to solve but an important constraint must be taken into account: the function $r$ is only defined for non-negative arguments and therefore we must obtain a solution satisfying $w_{i} \leq b_{i}$. This condition makes the problem more delicate.

In order to give a mathematical scope to the chemical attack problem, we introduce different functional spaces ([10] and [11]). We denote by $\Omega$ the set $] 0, \infty\left[\right.$ and by $Q_{T}$ the set $] 0, T[\times \Omega$. Let $X$ be a Banach space, we shall use the spaces $L^{2}(0, T ; X)$ and $C([0, T] ; X)$ endowed with their usual norm. We recall also the definitions

$$
\begin{aligned}
& V_{2}^{1,0}\left(Q_{T}\right) \stackrel{\text { def }}{=}\left\{\psi \in L^{2}\left(0, T ; H^{1}(\Omega)\right) ; \psi \in C\left([0, T] ; L^{2}(\Omega)\right)\right\}, \\
& W_{2}^{1,1}\left(Q_{T}\right) \stackrel{\text { def }}{=}\left\{\psi \in L^{2}\left(0, T ; H^{1}(\Omega)\right) ; \partial_{t} \psi \in L^{2}\left(0, T ; H^{-1}(\Omega)\right)\right\} .
\end{aligned}
$$

From an interpolation result [11, Lemma 11], we have the embedding

$$
\left(L^{\infty}\left(0, T ; L^{2}(\Omega)\right), L^{2}\left(0, T ; H^{1}(\Omega)\right)\right)_{\theta, 2} \subset L^{p}\left(0, T ; H^{\theta}(\Omega)\right)
$$

with $p=2 / \theta, \theta \in] 0,1[$. Applying property (3.1) with $\theta>1 / 2$, we find

$$
V_{2}^{1,0}\left(Q_{T}\right) \subset L^{p}\left(0, T ; C^{0}(\bar{\Omega})\right) .
$$

Consequently, taking $\theta=2 / 3$, the trace operator is continuous from $V_{2}^{1,0}\left(Q_{T}\right)$ into $L^{3}(0, T)$ and gives the estimate

$$
\|\phi(t, 0)\|_{L^{3}(0, T)} \leq\|\phi\|_{L^{3}\left(0, T ; C^{0}(\bar{\Omega})\right)} \leq c_{2}\|\phi\|_{V_{2}^{1,0}(\Omega)}
$$

where $c_{2}$ does not depend on $\phi$. Using the Hölder inequality, we draw the estimate

$$
\begin{aligned}
\|\phi(t, 0)\|_{L^{2}(0, T)} & \leq c_{2} T^{\frac{1}{6}}\|\phi(t, 0)\|_{L^{3}(0, T)} \\
& \leq c_{1}\|\phi\|_{V_{2}^{1,0}\left(Q_{T}\right)},
\end{aligned}
$$

where the constant $c_{1}$ does not depend on $\phi$. 
We propose a proof of existence and uniqueness in two steps. Assume first that $w_{i}^{0} \in L^{2}(\Omega) \cap L^{\infty}(\Omega)$, we shall prove the existence of a unique local solution in space $W_{2}^{1,1}\left(Q_{\delta}\right) \cap L^{\infty}\left(Q_{\delta}\right)$ for a $\left.\left.\delta \in\right] 0, T\right]$ small enough with $w_{i} \leq b_{i}$. To this end, we introduce a continuous non-linear operator $\Phi$ defined on a closed convex subspace $S(\delta)$ of $H^{1}(0, \delta)$

$$
S(\delta)=\left\{\alpha \in H^{1}(0, \delta) ; \alpha(0)=0 \text { with } 0 \geq \alpha^{\prime} \geq-K\right\}
$$

where $K$ is given by

$$
K=\sup _{\xi_{i} \in\left[0, \max \left(\sup _{\Omega} u_{i}^{0}, a_{i}\right)\right]} r\left(\xi_{1}, \xi_{2}, \xi_{3}\right) .
$$

such that the fixed points of $\Phi$ give the existence of solution for problem $\mathcal{P}_{\sqsupseteq}$.

Remark 3.2. One can note that the semi-norm $\left\|\alpha^{\prime}\right\|_{L^{2}(0, \delta)}$ is a norm for $S(\delta)$ since $\alpha(0)=0$.

Operator $\Phi$ is defined in the following way. Let $\alpha \in S(\delta)$ and assume there exists a solution for the linearised problem: find $\left(w_{i}\right)_{i=1}^{3}$ in $W_{2}^{1,1}\left(Q_{\delta}\right) \cap$ $L^{\infty}\left(Q_{\delta}\right)$ with $w_{i} \leq b_{i}$ such that for almost every $\left.t \in\right] 0, \delta$ [ we have

$$
\begin{array}{r}
\int_{0}^{\infty} \partial_{t} w_{i} \phi d y-\int_{0}^{\infty} \alpha^{\prime} \partial_{y} w_{i} \phi d y+D \int_{0}^{\infty} \partial_{y} w_{i} \partial_{y} \phi d y \\
=\alpha^{\prime}(t)\left(a_{i}-b_{i}+w_{i}(t, 0)\right) \phi(0) \quad \forall \phi \in H^{1}(\Omega) .
\end{array}
$$

Then we set with $v_{i}=b_{i}-w_{i}$

$$
\widetilde{\alpha}(t)=-\int_{0}^{t} r\left(v_{1}(\tau, 0), v_{2}(\tau, 0), v_{3}(\tau, 0)\right) d \tau .
$$

Let $\Phi$ be the operator $\alpha \mapsto \widetilde{\alpha}=\Phi(\alpha)$. We shall prove that $\Phi$ is well-defined and is a contraction mapping in $S(\delta)$ for a small enough $\delta \in] 0, T]$. We obtain then the local existence and uniqueness of the solution for problem $\mathcal{P}_{\sqsupseteq}$.

In a second step, we shall see that the solution can be extended to the whole interval $[0, T]$. We do the proof assuming $w_{i}^{0} \in H^{1}(\Omega)$ for the sake of simplicity but the result holds if $w_{i}^{0} \in L^{2}(\Omega)$. The main argument is based on the fact that the rate consumption tends to zero when the reactive concentrations (in our case $v_{2}$ and $v_{3}$ ) tend to zero (see relation 2.6). This property yields that functions $v_{i}$ remain non-negative (i.e. $w_{i} \leq b_{i}$ ).

In the next section, we present some technical lemmas. In the last section we prove the existence and the uniqueness of the solution for a small time $\delta$. Using the property of $r$ (see Remark 2.1), we prove that the solution can be extended on $[0, T]$ for every $T>0$. 


\section{Some technical lemmas}

In this section we study the linearised problem. We present here a serie of lemmas we shall use in the next sections to prove the existence and the uniqueness of the solution of problem $\mathcal{P}_{\sqsupset}$ on $Q_{\delta}$ for a small enough $\delta$. Let $a$ and $b$ be two real numbers with $b \geq 0$ and $w^{0} \in L^{2}(\Omega) \cap L^{\infty}(\Omega)$ satisfying $\nu \stackrel{\text { def }}{=} \max _{\Omega} w^{0} \leq b$. For any function $\alpha \in S(\delta), \delta>0$, we consider the following linear problem: find $w \in W_{2}^{1,1}\left(Q_{\delta}\right) \cap L^{\infty}\left(Q_{\delta}\right)$ such that for almost $t \in] 0, \delta[$ we have

$$
\begin{array}{cc}
\int_{0}^{\infty} \partial_{t} w \phi d y-\int_{0}^{\infty} \alpha^{\prime} \partial_{y} w \phi d y+\int_{0}^{\infty} \partial_{y} w \partial_{y} \phi d y \\
=\alpha^{\prime}(t)(a-b+w(t, 0)) \phi(0) & \forall \phi \in H^{1}(\Omega), \\
w(0, y)=w^{0}(y) & \text { on } \Omega .
\end{array}
$$

Remark 4.1. We present the results assuming that $D=1$ for the sake of simplicity. The general case (i.e. $D>0$ ) derives easily from this particular case.

Lemma 4.1. Let $\alpha \in S(\delta)$, then there exists a unique solution $w$ in $W_{2}^{1,1}\left(Q_{\delta}\right)$ for problem (4.1). Furthermore, we have the estimate

$$
\|w\|_{V_{2}^{1,0}\left(Q_{\delta}\right)} \leq C_{1}\left(K \sqrt{\delta}+\left\|w^{0}\right\|_{L^{2}(\Omega)}\right)
$$

where the constant $C_{1}$ does not depend on $\alpha, \delta$ and $K$.

Proof. Existence and uniqueness of the solution $w \in W_{2}^{1,1}\left(Q_{\delta}\right)$ can be obtained using classical tools (see for instance [3, p. 218] or [10]). Taking $\phi=w$ in relation (4.1) and integrating in time on $[0, \tau], \tau \leq \delta$, we obtain

$$
\begin{gathered}
\int_{0}^{\infty} \int_{0}^{\tau} \frac{1}{2} \partial_{t}\left(w^{2}\right) d y d t-\int_{0}^{\infty} \int_{0}^{\tau} \frac{\alpha^{\prime}}{2} \partial_{y}\left(w^{2}\right) d y d t+\int_{0}^{\infty} \int_{0}^{\tau}\left(\partial_{y} w\right)^{2} d y d t \\
=\int_{0}^{\tau} \alpha^{\prime}(t)(a-b) w(t, 0) d t+\int_{0}^{\tau} \alpha^{\prime}(t) w^{2}(t, 0) d t .
\end{gathered}
$$

Since we have $\alpha^{\prime} \leq 0$, the following inequality holds

$$
\begin{aligned}
& \frac{1}{2}\|w(\tau)\|_{L^{2}(\Omega)}^{2}+\int_{0}^{\infty} \int_{0}^{\tau}\left(\partial_{y} w\right)^{2} d y d t \\
& \quad=\int_{0}^{\tau} \alpha^{\prime}(t)(a-b) w(t, 0) d t+\frac{1}{2}\left\|w^{0}\right\|_{L^{2}(\Omega)}^{2}+\frac{1}{2} \int_{0}^{\tau} \alpha^{\prime}(t) w^{2}(t, 0) d t \\
& \quad \leq \int_{0}^{\tau} \alpha^{\prime}(t)(a-b) w(t, 0) d t+\frac{1}{2}\left\|w^{0}\right\|_{L^{2}(\Omega)}^{2} .
\end{aligned}
$$


We can write using relations (3.3)

$$
\begin{aligned}
\frac{1}{2}\|w(\tau)\|_{L^{2}(\Omega)}+\left\|\partial_{y} w\right\|_{L^{2}\left(Q_{\tau}\right)}^{2} & \leq K \sqrt{\tau}|a-b|\|w(t, 0)\|_{L^{2}(0, \tau)}+\frac{1}{2}\left\|w^{0}\right\|_{L^{2}(\Omega)}^{2} \\
& \leq K \sqrt{\delta}|a-b|\|w(t, 0)\|_{L^{2}(0, \delta)}+\frac{1}{2}\left\|w^{0}\right\|_{L^{2}(\Omega)}^{2} \\
& \leq c_{1} \sqrt{\delta} K|a-b|\|w\|_{V_{2}^{1,0}\left(Q_{\delta}\right)}+\frac{1}{2}\left\|w^{0}\right\|_{L^{2}(\Omega)}^{2} \\
& \leq\left(c_{1} \sqrt{\delta} K|a-b|\right)^{2}+\frac{1}{4}\|w\|_{V_{2}^{1,0}\left(Q_{\delta}\right)}^{2} \\
& +\frac{1}{2}\left\|w^{0}\right\|_{L^{2}(\Omega)}^{2} .
\end{aligned}
$$

Thus, there exists a constant $C$ such that for all $\tau \leq \delta$ we have

$$
\begin{aligned}
& \frac{1}{2}\|w(\tau)\|_{L^{2}(\Omega)}^{2}+\left\|\partial_{y} w\right\|_{L^{2}\left(Q_{\tau}\right)}^{2} \\
& \quad \leq C^{2}\left(K^{2} \delta|a-b|^{2}+\left\|w^{0}\right\|_{L^{2}(\Omega)}^{2}\right)+\frac{1}{4}\|w\|_{V_{2}^{1,0}\left(Q_{\delta}\right)}^{2} .
\end{aligned}
$$

Taking the upper bound for $\tau$ in relation (4.3), we obtain

$$
\begin{aligned}
& \frac{1}{2}\|w\|_{L^{\infty}\left(0, \delta, L^{2}(\Omega)\right)}^{2}+\left\|\partial_{y} w\right\|_{L^{2}\left(Q_{\delta}\right)}^{2} \\
& \quad \leq C^{2}\left(K^{2} \delta|a-b|^{2}+\left\|w^{0}\right\|_{L^{2}(\Omega)}^{2}\right)+\frac{1}{4}\|w\|_{V_{2}^{1,0}\left(Q_{\delta}\right)}^{2} .
\end{aligned}
$$

From the last inequality, we draw relation (4.2) and the lemma is proved.

Lemma 4.2. Functions $w \in L^{\infty}\left(Q_{\delta}\right)$ with the estimate

$$
w(t, y) \in\left[\min \left(b-a, \inf _{\Omega} w^{0}\right), \max \left(b-a, \sup _{\Omega} w^{0}\right)\right]
$$

$$
\text { a.e. }(t, y) \in Q_{\delta} \text {. }
$$

Proof. We use the Stampacchia truncature method [3, p. 189]. For $\psi$ measurable function, we denote by $[\psi]^{+}$the non-negative part of $\psi$ and by $[\psi]^{-}$the non-positive part. Let $k$ be given by

$$
k=\max \left(b-a, \sup _{\Omega} w^{0}\right) \geq 0,
$$

we have $\phi=[w-k]^{+} \in L^{2}\left(0, \delta ; H^{1}(\Omega)\right)$. Using relation (4.1), we can write

$$
\begin{aligned}
\int_{0}^{\infty} & \int_{0}^{\delta} \frac{1}{2} \partial_{t}\left(\left([w-k]^{+}\right)^{2}\right) d y d t-\int_{0}^{\infty} \int_{0}^{\delta} \frac{\alpha^{\prime}}{2} \partial_{y}\left(\left([w-k]^{+}\right)^{2}\right) d y d t+ \\
& \int_{0}^{\infty} \int_{0}^{\delta}\left(\partial_{y}[w-k]^{+}\right)^{2} d y d t=\int_{0}^{\delta} \alpha^{\prime}(t)(a-b+w(t, 0))[w(t, 0)-k]^{+} d t .
\end{aligned}
$$


Since $k \geq \sup _{\Omega} w^{0}$, we have $\left[w^{0}-k\right]^{+}=0$ and we get

$$
\begin{aligned}
& \int_{0}^{\infty} \frac{1}{2}\left([w(\delta)-k]^{+}\right)^{2} d y+\int_{0}^{\infty} \int_{0}^{\delta}\left(\partial_{y}[w-k]^{+}\right)^{2} d y d t \\
& =\int_{0}^{\delta} \alpha^{\prime}(t)\left(a-b+w(t, 0)-\frac{1}{2}[w(t, 0)-k]^{+}\right)[w(t, 0)-k]^{+} d t .
\end{aligned}
$$

if $k \geq b-a$, the quantity

$$
\alpha^{\prime}(t)\left(a-b+w(t, 0)-\frac{1}{2}[w(t, 0)-k]^{+}\right)[w(t, 0)-k]^{+}
$$

is non-positive therefore we have $\left\|\partial_{y}[w-k]^{+}\right\|_{L^{2}\left(Q_{\delta}\right)} \leq 0$, hence $w \leq k$.

Using the function $\phi=[w-k]^{-}$with $k=\min \left(b-a, \inf _{\Omega} w^{0}\right)$, we obtain the lower bound.

Remark 4.2. The relation (4.5) remains valid under the unique assumption $k \geq \nu$.

Remark 4.3. Notice that Lemma 4.2 implies that the function $t \mapsto w(t, 0) \in$ $L^{\infty}(0, \delta)$ and satisfies also relation (4.4).

Remark 4.4. Since $\nu \leq b$, the condition $a \geq 0$ implies immediately $w \leq b$ on $Q_{\delta}$. The problem arises when $a<0$. This is the difficult part of the problem and we need a more accurate estimate for $w$.

In order to give a sharp estimate for the upper bound of $w$, we recall the following result one can find in [12, p. 93].

Lemma 4.3. Let $\lambda(k)$ be a non-negative non-increasing function defined on $\left[k_{0}, \infty\left[, k_{0} \in \mathbb{R}\right.\right.$ and assume that there exists constants $\gamma>0$ and $\beta>1$ such that for $k_{0} \leq k<k^{\prime}$ we have

$$
\lambda\left(k^{\prime}\right) \leq\left(\frac{c}{k^{\prime}-k}\right)^{\gamma} \lambda^{\beta}(k)
$$

where $c$ does not depend on $k$ and $k^{\prime}$. Then $\lambda\left(k_{0}+d\right)=0$ with

$$
d=\left(\lambda\left(k_{0}\right)\right)^{(\beta-1) / \alpha} 2^{\beta /(\beta-1)} c .
$$

We now prove that the assumption $\nu<b$ implies the existences of $\delta>0$ such that $w \leq b$ on $Q_{\delta}$.

Lemma 4.4. Let $\delta>0$, we have the following estimate

$$
\left\|[w-\nu]^{+}\right\|_{V_{2}^{1,0}\left(Q_{\delta}\right)} \leq C_{1} K \sqrt{\delta} .
$$


Proof. We introduce the function $\phi=[w-\nu]^{+}$in relation (4.1). As we do in Lemma 4.1, we draw for the function $[w-\nu]^{+}$a relation similar to the relation (4.2). Noting that $\left[w^{0}-\nu\right]^{+}=0$ and we get the estimate (4.7).

Lemma 4.5. Assume $\nu<b$ then there exists $\left.\left.\delta_{1} \in\right] 0, T\right]$ such that $w \leq$ $\nu+(b-\nu) / 2$ on $Q_{\delta_{1}}$.

Proof. Let $\delta, k$ be non-negative real numbers and $\tau \in] 0, \delta]$, we denote by $B_{k}(\tau)$ the set $B_{k}(\tau)=\left\{(t, x) \in Q_{\tau} ; w>k\right\}$. Let $k^{\prime}>k \geq \nu$, we can write using $[10$, p. 75$]$

$$
\begin{aligned}
\left(k^{\prime}-k\right)\left|B_{k^{\prime}}(\delta)\right|^{\frac{1}{4}} & \leq\left\|[w-k]^{+}\right\|_{L^{4}\left(Q_{\delta}\right)} \\
& \leq \beta\left\|[w-k]^{+}\right\|_{V_{2}^{1,0}\left(Q_{\delta}\right)}
\end{aligned}
$$

where $\beta$ does not depend on $w, \delta$ and $k$. On the other hand, relation (4.5) gives for every $\tau \in[0, \delta]$

$$
\begin{aligned}
\int_{0}^{\infty} & \frac{1}{2}\left([w(\tau)-k]^{+}\right)^{2} d y+\int_{0}^{\infty} \int_{0}^{\tau}\left(\partial_{y}[w-k]^{+}\right)^{2} d y d t \\
& =\int_{0}^{\tau} \alpha^{\prime}(t)\left(a-b+w(t, 0)-\frac{1}{2}[w-k]^{+}\right)[w-k]^{+} d t \\
& =\int_{0}^{\infty} \int_{0}^{\tau} \alpha^{\prime}(t) \partial_{y}\left\{\left(a-b+w-\frac{1}{2}[w-k]^{+}\right)[w-k]^{+}\right\} d t d y \\
& =\int_{0}^{\infty} \int_{0}^{\tau} \alpha^{\prime}(t)(a-b+w) \partial_{y}\left([w-k]^{+}\right) d t d y \\
& \leq\left\|\alpha^{\prime}(t)(a-b+w)\right\|_{L^{2}\left(B_{k}(\tau)\right)}\left\|\partial_{y}\left([w-k]^{+}\right)\right\|_{L^{2}\left(B_{k}(\tau)\right)} .
\end{aligned}
$$

Hence, we get

$$
\begin{gathered}
\int_{0}^{\infty} \frac{1}{2}\left([w(\tau)-k]^{+}\right)^{2} d y+\int_{0}^{\infty} \int_{0}^{\tau} \frac{1}{2}\left(\partial_{y}[w-k]^{+}\right)^{2} d y d t \\
\leq \frac{1}{2}\left\|\alpha^{\prime}(a-b+w)\right\|_{L^{2}\left(B_{k}(\tau)\right)}^{2}
\end{gathered}
$$

Since $w \leq b-a$, we can write

$$
\begin{gathered}
\int_{0}^{\infty} \frac{1}{2}\left([w(\tau)-k]^{+}\right)^{2} d y+\int_{0}^{\infty} \int_{0}^{\tau} \frac{1}{2}\left(\partial_{y}[w-k]^{+}\right)^{2} d y d t \\
\leq C^{2} K^{2}(b-a)^{2}\left|B_{k}(\tau)\right| .
\end{gathered}
$$

Using the fact that $\left|B_{k}(\tau)\right| \leq\left|B_{k}(\delta)\right|$ and taking the upper bound for $\tau \leq \delta$, we draw from the last relation the estimate

$$
\left\|[w-k]^{+}\right\|_{V_{2}^{1,0}\left(Q_{\delta}\right)}^{2} \leq C^{2} K^{2}(b-a)^{2}\left|B_{k}(\delta)\right|
$$


and we get from relation (4.8)

$$
\left(k^{\prime}-k\right)^{4}\left|B_{k^{\prime}}(\delta)\right| \leq C^{\prime} K^{4}\left|B_{k}(\delta)\right|^{2} .
$$

Constant $C^{\prime}$ does not depend on $k^{\prime}, k, \delta$ and $K$. Applying Lemma 4.3 with $\lambda(k)=\left|B_{k}(\delta)\right|$ and $k_{0}=\nu+(b-\nu) / 4$, the previous inequality yields

$$
\left|B_{\nu+(b-\nu) / 4+d}(\delta)\right|=0
$$

with

$$
d=C^{\prime \prime} K\left|B_{\nu+(b-\nu) / 4}(\delta)\right|^{1 / 4},
$$

where $C^{\prime \prime}$ does not depend on $\delta$ and $K$. Hence we get

$$
\sup _{Q_{\delta}} w \leq \nu+\frac{b-\nu}{4}+C^{\prime \prime} K\left|B_{\nu+(b-\nu) / 4}(\delta)\right|^{1 / 4} .
$$

Using relation (4.8) with $k=\nu$ and $k^{\prime}=\nu+(b-\nu) / 4$, we draw the inequality

$$
\frac{b-\nu}{4}\left|B_{\nu+(b-\nu) / 4}(\delta)\right|^{1 / 4} \leq \beta\left\|[w-\nu]^{+}\right\|_{V_{2}^{1,0}\left(Q_{\delta}\right)} .
$$

Relation (4.7) gives the following estimate

$$
\left\|[w-\nu]^{+}\right\|_{V_{2}^{1,0}\left(Q_{\delta}\right)} \leq C_{1} K \sqrt{\delta}
$$

hence we get

$$
\left|B_{\nu+(b-\nu) / 4}(\delta)\right|^{1 / 4} \leq \beta 4 C_{1} \frac{K \sqrt{\delta}}{b-\nu}
$$

thus

$$
\sup _{Q \delta} w \leq \nu+\frac{b-\nu}{4}+\widetilde{C} \frac{K^{2} \sqrt{\delta}}{b-\nu} .
$$

The constant $\widetilde{C}$ does not depend on $\delta$ and $K$. Taking $\delta_{1}$ such that

$$
\widetilde{C} \frac{K^{2} \sqrt{\delta}_{1}}{b-\nu} \leq \frac{b-\nu}{4}
$$

and we get $w \leq \nu+(b-\nu) / 2$ on $Q_{\delta_{1}}$.

Lemma 4.6. The mapping $\alpha \mapsto w$ is continuous from $S(\delta)$ into $V_{2}^{1,0}\left(Q_{\delta}\right)$. Furthermore, let $\alpha, \widetilde{\alpha} \in S(\delta)$ and $w, \widetilde{w}$ the associated solutions, we have the estimate

$$
\|w-\widetilde{w}\|_{V_{2}^{1,0}\left(Q_{\delta}\right)} \leq C_{2}\left\|\alpha^{\prime}-\widetilde{\alpha}^{\prime}\right\|_{L^{2}(0, \delta)} .
$$

Constant $C_{2}$ does not depend on $\alpha, w, \widetilde{\alpha}, \widetilde{w}$. 
Proof. Let $w^{0}$ and $b$ be fixed, we consider a function $\alpha$ in $S(\delta)$ and $w$ the solution in $W_{2}^{1,1}\left(Q_{\delta}\right) \cap L^{\infty}\left(Q_{\delta}\right)$ of problem (3.4) (resp. $\widetilde{\alpha}$ and $\widetilde{w}$ ). We have for all $\phi \in H^{1}(\Omega)$

$$
\begin{aligned}
& \int_{0}^{\infty} \partial_{t}(w-\widetilde{w}) \phi d y-\int_{0}^{\infty} \alpha^{\prime} \partial_{y}(w-\widetilde{w}) \phi d y \\
& -\int_{0}^{\infty}\left(\alpha^{\prime}-\widetilde{\alpha}^{\prime}\right) \partial_{y} \widetilde{w} \phi d y+\int_{0}^{\infty} \partial_{y}(w-\widetilde{w}) \partial_{y} \phi d y \\
& =\left(\alpha^{\prime}-\widetilde{\alpha}^{\prime}\right)(a-b) \phi(0)+(w(t, 0)-\widetilde{w}(t, 0)) \alpha^{\prime} \phi(0)+\left(\alpha^{\prime}-\widetilde{\alpha}^{\prime}\right) \widetilde{w}(t, 0) \phi(0)
\end{aligned}
$$

Taking $\phi=w-\widetilde{w}$ and integrating in time on $[0, \tau], \tau \in[0, \delta]$, we obtain

$$
\begin{aligned}
& \int_{0}^{\infty} \int_{0}^{\tau} \frac{1}{2} \partial_{t}(w-\widetilde{w})^{2} d y d t-\int_{0}^{\infty} \int_{0}^{\tau} \frac{1}{2} \alpha^{\prime} \partial_{y}(w-\widetilde{w})^{2} d y d t \\
& \quad+\int_{0}^{\infty} \int_{0}^{\tau}\left(\partial_{y}(w-\widetilde{w})\right)^{2} d y d t=\int_{0}^{\infty} \int_{0}^{\tau}\left(\alpha^{\prime}-\widetilde{\alpha}^{\prime}\right) \partial_{y} \widetilde{w}(w-\widetilde{w}) d y d t \\
& \quad+\int_{0}^{\tau}(w(t, 0)-\widetilde{w}(t, 0))^{2} \alpha^{\prime} d t+\int_{0}^{\tau}\left(\alpha^{\prime}-\widetilde{\alpha}^{\prime}\right)(a-b)(w(t, 0)-\widetilde{w}(t, 0)) d t \\
& \quad+\int_{0}^{\tau}\left(\alpha^{\prime}-\widetilde{\alpha}^{\prime}\right) \widetilde{w}(t, 0)(w(t, 0)-\widetilde{w}(t, 0)) d t
\end{aligned}
$$

Using the facts that $\alpha^{\prime} \leq 0$ and $(w-\widetilde{w})(0, \cdot)=0$, we simplify the relation

$$
\begin{aligned}
\frac{1}{2} \| w(\tau)- & \left.\widetilde{w}(\tau)\left\|_{L^{2}(\Omega)}^{2}+\right\| \partial_{y}(w-\widetilde{w})\right) \|_{L^{2}\left(Q_{\tau}\right)}^{2} \\
& \leq \int_{0}^{\infty} \int_{0}^{\tau}\left|\alpha^{\prime}-\widetilde{\alpha}^{\prime}\right|\left|(w-\widetilde{w}) \partial_{y} \widetilde{w}\right| d y d t \\
& +\int_{0}^{\tau}\left|\alpha^{\prime}-\widetilde{\alpha}^{\prime}\right||(a-b)(w(t, 0)-\widetilde{w}(t, 0))| d t \\
& +\int_{0}^{\tau}\left|\alpha^{\prime}-\widetilde{\alpha}^{\prime}\right||\widetilde{w}(t, 0)(w(t, 0)-\widetilde{w}(t, 0))| d t .
\end{aligned}
$$

Thanks to the Cauchy-Schwarz inequality we write

$$
\begin{aligned}
& \left.\frac{1}{2}\|w(\tau)-\widetilde{w}(\tau)\|_{L^{2}(\Omega)}^{2}+\| \partial_{y}(w-\widetilde{w})\right) \|_{L^{2}\left(Q_{\tau}\right)}^{2} \\
& \leq\left\|\alpha^{\prime}-\widetilde{\alpha}^{\prime}\right\|_{L^{2}(0, \tau)}\left\|\partial_{y} \widetilde{w}\right\|_{L^{2}\left(Q_{\tau}\right)}\|w-\widetilde{w}\|_{L^{\infty}\left(0, \tau ; L^{2}(\Omega)\right)} \\
& \quad+\left\|\alpha^{\prime}-\widetilde{\alpha}^{\prime}\right\|_{L^{2}(0, \tau)}|a-b|\|(w-\widetilde{w})(\cdot, 0)\|_{L^{2}(0, \tau)} \\
& \quad+\left\|\alpha^{\prime}-\widetilde{\alpha}^{\prime}\right\|_{L^{2}(0, \tau)}\|\widetilde{w}(\cdot, 0)\|_{L^{\infty}(0, \tau)}\|(w-\widetilde{w})(\cdot, 0)\|_{L^{2}(0, \tau)}
\end{aligned}
$$


Using estimates (3.3) and (4.2), we draw the inequality

$$
\begin{aligned}
& \left.\frac{1}{2}\|w(\tau)-\widetilde{w}(\tau)\|_{L^{2}(\Omega)}^{2}+\| \partial_{y}(w-\widetilde{w})\right) \|_{L^{2}\left(Q_{\tau}\right)}^{2} \\
& \leq 4\left\|\alpha^{\prime}-\widetilde{\alpha}^{\prime}\right\|_{L^{2}(0, \tau)}\left(C_{1} \sqrt{\tau} K+C_{1}\left\|w^{0}\right\|_{L^{2}(\Omega)}^{2}+|a-b| c_{1}+c_{1}\|\widetilde{w}\|_{L^{\infty}\left(Q_{\tau}\right)}\right)^{2} \\
& \quad+\frac{1}{4}\|w-\widetilde{w}\|_{V_{2}^{1,0}\left(Q_{\tau}\right)} .
\end{aligned}
$$

Since $\tau \leq \delta$, we obtain the estimate

$$
\begin{aligned}
& \left.\frac{1}{2}\|w(\tau)-\widetilde{w}(\tau)\|_{L^{2}(\Omega)}^{2}+\| \partial_{y}(w-\widetilde{w})\right) \|_{L^{2}\left(Q_{\tau}\right)}^{2} \\
& \leq 4\left\|\alpha^{\prime}-\widetilde{\alpha}^{\prime}\right\|_{L^{2}(0, \delta)}^{2}\left(C_{1} \sqrt{\delta} K+C_{1}\left\|w^{0}\right\|_{L^{2}(\Omega)}+|a-b| c_{1}+c_{1}\|\widetilde{w}\|_{L^{\infty}\left(Q_{\delta}\right)}\right)^{2} \\
& \quad+\frac{1}{4}\|w-\widetilde{w}\|_{V_{2}^{1,0}\left(Q_{\delta}\right)} .
\end{aligned}
$$

Taking the upper bound for $\tau$ as we do in Lemma 4.1 and the estimate (4.13) is proved.

\section{Existence and uniqueness of the global solution}

We first prove the local existence and uniqueness of the solution for problem $\mathcal{P}_{\sqsupseteq}$. We consider the non-linear operator $\Phi$ on $S(\delta)$ we introduce in Section 3 and prove the existence of a fixed point. Let $\alpha \in S(\delta)$, Lemma 4.1 gives a unique solution $\left(w_{1}, w_{2}, w_{3}\right)$ in $W_{2}^{1,1}\left(Q_{\delta}\right)$ for problem (3.4). Lemma 4.2 shows that the functions $w_{i}$ are bounded. Furthermore, since $a_{1}$ is nonnegative, estimate (4.4) yields that $w_{1} \leq b_{1}$ but a priori we have not this property for $w_{2}$ and $w_{3}$.

We prove in Lemma 4.5 that assuming $\nu_{2}<b_{2}$ and $\nu_{3}<b_{3}$, there exists $\delta_{1}>0$ such that $w_{2} \leq b_{2}$ and $w_{3} \leq b_{3}$ on $Q_{\delta_{1}}$. In that case, the functions $v_{i}=b_{i}-w_{i}, i=1,2,3$ are non-negative on $Q_{\delta_{1}}$ and operator $\Phi$ is well-defined on $S\left(\delta_{1}\right)$. One can check that estimate (4.4) yields that $\Phi(\alpha) \in S\left(\delta_{1}\right)$ for all $\alpha \in S\left(\delta_{1}\right)$. The following lemma shows that operator $\Phi$ defines a contraction in $S(\delta)$ for a $\delta \in] 0, \delta_{1}\left[\right.$ small enough. To this end, let $w_{i}^{0} \in L^{2}(\Omega) \cap L^{\infty}(\Omega)$ and $b_{i}$ be fixed, we consider two elements $\alpha, \widetilde{\alpha}$ of $S(\delta)$ and we denote by $\left(w_{i}\right)_{i=1}^{3},\left(\widetilde{w}_{i}\right)_{i=1}^{3}$ in $W_{2}^{1,1}\left(Q_{\delta_{1}}\right) \cap L^{\infty}\left(Q_{\delta_{1}}\right)$ the respective solutions of the problem (3.4).

Lemma 5.1. For all $\delta \in] 0, \delta_{1}$ ], we have the estimate

$$
\left\|(\Phi(\alpha))^{\prime}-(\Phi(\widetilde{\alpha}))^{\prime}\right\|_{L^{2}(0, \delta)} \leq C_{3} \delta^{\frac{1}{6}}\left\|\alpha^{\prime}-\widetilde{\alpha}^{\prime}\right\|_{L^{2}(0, \delta)}
$$

where $C_{3}$ does not depend on $\delta$. 
Proof. Let $v_{i}=b_{i}-w_{i}, \widetilde{v}_{i}=b_{i}-\widetilde{w}_{i}$, since $w_{i}$ and $\widetilde{w}_{i}$ are bounded solutions for the problem $\mathcal{P}_{\sqsupseteq}$ we have using the Lipschitz property of $r$

$$
\begin{aligned}
\left\|(\Phi(\alpha))^{\prime}-(\Phi(\widetilde{\alpha}))^{\prime}\right\|_{L^{2}(0, \delta)} & \leq \delta^{\frac{1}{6}}\left\|(\Phi(\alpha))^{\prime}-(\Phi(\widetilde{\alpha}))^{\prime}\right\|_{L^{3}(0, \delta)} \\
& \leq \delta^{\frac{1}{6}} L \sum_{i=1}^{3}\left\|w_{i}(t, 0)-\widetilde{w}_{i}(t, 0)\right\|_{L^{3}(0, \delta)}
\end{aligned}
$$

where the constant $L$ depends only on $a_{i}, b_{i}$ and $\left\|w_{i}^{0}\right\|_{L^{\infty}(\Omega)}$. Relation (3.2) and Lemma 4.6 yield

$$
\begin{aligned}
\left\|(\Phi(\alpha))^{\prime}-(\Phi(\widetilde{\alpha}))^{\prime}\right\|_{L^{2}(0, \delta)} & \leq c_{2} L \delta^{\frac{1}{6}} \sum_{i=1}^{3}\left\|w_{i}-\widetilde{w}_{i}\right\|_{V_{2}^{1,0}(0, \delta)} \\
& \leq 3 c_{2} C_{2} L \delta^{\frac{1}{6}}\left\|\alpha^{\prime}-\widetilde{\alpha}^{\prime}\right\|_{L^{2}(0, \delta)}
\end{aligned}
$$

and the lemma is proved.

Theorem 5.1 (Local existence and uniqueness). If $\nu_{2}<b_{2}$ and $\nu_{3}<b_{3}$, then there exists $\delta$ such that the problem $\mathcal{P}_{\sqsupseteq}$ has a unique solution $\left(w_{i}\right)_{i=1}^{3}$ in $W_{2}^{1,1}\left(Q_{\delta}\right) \cap L^{\infty}\left(Q_{\delta}\right)$. Furthermore, assuming $w_{i}^{0} \in H^{1}(\Omega)$, functions $w_{i} \in C^{0}\left(\bar{Q}_{\delta}\right)$.

Proof. Let $\delta \in] 0, \delta_{1}$ [, operator $\Phi$ is well-defined from $S(\delta)$ into itself. Choosing $\delta$ such that $3 c_{2} C_{2} L \delta^{\frac{1}{6}}<1$, Lemma 5.1 yields that the operator $\Phi$ is a mapping contraction, hence there exists a unique fixed point $\alpha$ in $S(\delta)$. Lemmas 4.1, 4.2 and 4.4 say that there exists a unique solution $\left(w_{i}\right)_{i=1}^{3}$ in $W_{2}^{1,1}\left(Q_{\delta}\right) \cap L^{\infty}\left(Q_{\delta}\right)$ for problem $\mathcal{P}_{\sqsupseteq}$ with $w_{1} \leq b_{1}, w_{2}<b_{2}, w_{3}<b_{3}$. Since the function $r\left(w_{1}(\cdot, 0), w_{2}(\cdot, 0), w_{3}(\cdot, 0)\right.$ is bounded and functions $w_{i}(\cdot, 0)$ belong to $L^{2}(0, T)$ with $w_{i}^{0} \in H^{1}(\Omega)$, we have $w_{i} \in L^{2}\left(0, T ; H^{2}(\Omega)\right)$ and $\partial_{t} w_{i} \in L^{2}\left(0, T ; L^{2}(\Omega)\right)$. Hence $w_{i} \in C^{0}\left(\bar{Q}_{\delta}\right)$.

Remark 5.1. If we just assume $w_{i}^{0} \in L^{2}(\Omega) \cap L^{\infty}(\Omega)$, we get $w_{i} \in$ $L^{2}\left(\varepsilon, T ; H^{2}(\Omega)\right)$ and $\partial_{t} w_{i} \in L^{2}\left(\varepsilon, T ; L^{2}(\Omega)\right)$ for every $\varepsilon>0$.

We now prove that the local solution in time given by Theorem 5.1 can be extended on the whole interval $[0, T]$ for every $T>0$. We denote by $\left.\left.T_{0} \in\right] 0, T\right]$ the unique real number defined by

$$
\left.T_{0}=\sup \{\delta \in] 0, T\right] ; \mathcal{P}_{\sqsupseteq} \text { has a solution on } \mathcal{Q}_{\delta} \text { with } \sqsupseteq_{\epsilon}<\left\lfloor_{\epsilon}, \sqsupseteq_{\ni}<\left\lfloor_{\ni}\right\}\right. \text {. }
$$

Theorem 5.1 gives the existence of $T_{0}$, we shall prove that $T_{0}=T$. To this end, assume $T_{0}<T$ and let $\left(w_{1}, w_{2}, w_{3}\right)$ an associated solution of problem $\mathcal{P}_{\sqsupseteq}$ on $Q_{T_{0}}$, we show that assumption $T_{0}<T$ yields a contradiction. 
Lemma 5.2. Let $\rho_{j}, j=2,3$ be the continuous non-decreasing functions defined on $\left[0, T_{0}\right]$ by

$$
\rho_{j}(\tau) \stackrel{\text { def }}{=} \sup _{Q_{\tau}} w_{j}
$$

Then one of the two equalities is satisfied

$$
\begin{aligned}
& \rho_{2}\left(T_{0}\right)=\sup _{\Omega} w_{2}\left(T_{0}, \cdot\right)=b_{2}, \\
& \rho_{3}\left(T_{0}\right)=\sup _{\Omega} w_{3}\left(T_{0}, \cdot\right)=b_{3} .
\end{aligned}
$$

Proof. Definition of $T_{0}$ yields that $\rho_{j}(\tau)<b_{j}$ for all $\tau \in\left[0, T_{0}[, j=2,3\right.$ hence by continuity of $\rho_{j}$, we have $\rho_{j}\left(T_{0}\right) \leq b_{j}$. Assume that $\rho_{j}\left(T_{0}\right)<b_{j}$, continuity of $w_{i}$ yields that we have $\sup _{\Omega} w_{i}\left(T_{0}, \cdot\right)<b_{i}$. We can then consider the problem $\mathcal{P}_{\sqsupseteq}$ with the new initial condition $w_{i}^{0}=w_{i}\left(T_{0}\right), i=1,2,3$. Theorem 5.1 gives existence of a $\delta>0$ such that problem $\mathcal{P}_{\sqsupseteq}$ admits a solution $\left(w_{i}\left(t+T_{0}, \cdot\right)\right), i=1,2,3$ on $Q_{\delta}$, hence we obtain a solution $\left(w_{1}, w_{2}, w_{3}\right)$ on $Q_{T_{0}+\delta}$ with $w_{i}<b_{i}, i=1,2,3$ which contradicts the definition of $T_{0}$. We cannot have both $w_{2}\left(T_{0}, \cdot\right)<b_{2}$ and $w_{3}\left(T_{0}, \cdot\right)<b_{3}$ on $\Omega$.

Remark 5.2. In the sequel we assume that the relation (5.3) is satisfied and $w_{2}<b_{2}$ on $Q_{T_{0}}$. The results are the same if we consider the relation (5.2) and $w_{3}<b_{3}$ on $Q_{T_{0}}$. The case where the two relations occur at the same time $T_{0}$ can be also treated in the same way.

Lemma 5.3. Let $\tau \in\left[0, T_{0}\right]$ and consider the continuous non-decreasing real functions $\gamma_{3}$ defined by

$$
\gamma_{3}(\tau) \stackrel{\text { def }}{=} \sup _{t \in[0, \tau]} w_{3}(t, 0) .
$$

There exists $T_{1} \in\left[0, T_{0}\left[\right.\right.$ satisfying $\rho_{3}\left(T_{1}\right)>\nu_{3}$ such that we have for all $\tau \in\left[T_{1}, T_{0}\right]$

$$
\rho_{3}(\tau)=\gamma_{3}(\tau) .
$$

In particular, we have $\gamma_{3}\left(T_{0}\right)=w_{3}\left(T_{0}, 0\right)=b_{3}$.

Proof. Remark 5.2 says that $\rho_{3}\left(T_{0}\right)=b_{3}$. Since $\nu_{3}<b_{3}$, the continuity of $\rho_{3}$ gives the existence of $T_{1}<T_{0}$ such that $\nu_{3}<\rho_{3}\left(T_{1}\right)<b_{3}$.

Let $\tau \in\left[T_{1}, T_{0}\left[\right.\right.$. Obviously we have $\gamma_{3}(\tau) \leq \rho_{3}(\tau)$. Assume $\gamma_{3}(\tau)<\rho_{3}(\tau)$ then the monotonicity of $\rho_{3}$ says that $\rho_{3}(\tau)>\nu_{3}$. We can choose a real number $k$ such that we have $\max \left(\gamma_{3}(\tau), \nu_{3}\right)<k<\rho_{3}(\tau)$. Let us consider 
the function $\phi=\left[w_{3}-k\right]^{+}$, since $k>\nu_{3}$, relation (4.5) is valid (see Remark 4.2) and we obtain

$$
\begin{aligned}
& \int_{0}^{\infty} \frac{1}{2}\left(\left[w_{3}(\tau)-k\right]^{+}\right)^{2} d y+\int_{0}^{\infty} \int_{0}^{\tau}\left(\partial_{y}\left[w_{3}-k\right]^{+}\right)^{2} d y d t \\
& \quad=\int_{0}^{\tau} \alpha^{\prime}(t)\left(a_{3}-b_{3}+w_{3}(t, 0)-\frac{1}{2}\left[w_{3}(t, 0)-k\right]^{+}\right)\left[w_{3}(t, 0)-k\right]^{+} d t
\end{aligned}
$$

The assumption $k>\gamma_{3}(\tau)$ yields $\left[w_{3}(t, 0)-k\right]^{+}=0$, hence we deduce

$$
\left\|\partial_{y}\left[w_{3}-k\right]^{+}\right\|_{L^{2}\left(Q_{\tau}\right)}^{2} \leq 0 .
$$

We get then $k \geq \rho_{3}(\tau)$ which refutes relation $k<\rho_{3}(\tau)$ and we obtain the relation (5.4). On the other hand, the definition of $T_{0}$ says that $\gamma_{3}(\tau)<b_{3}$ for $\tau<T_{0}$. Relation (5.4) and Lemma 5.2 yield that $\gamma_{3}\left(T_{0}\right)=b_{3}$, hence from the continuity of $w_{3}$ we draw the equality $w_{3}\left(T_{0}, 0\right)=b_{3}$ and the lemma is proved.

Lemma 5.4. Assume $T_{0}<T$, then we have $w_{3}\left(T_{0}, 0\right)<b_{3}$.

Proof. For $k$ real number, we denote by $A_{k}\left(T_{0}\right)$ the subset of $\left[0, T_{0}\right]$ given by $A_{k}\left(T_{0}\right)=\left\{t \in\left[0, T_{0}\right] ; w_{3}(t, 0) \geq k\right\}$. We can easily check that the function $k \mapsto\left|A_{k}\left(T_{0}\right)\right|$ is a continuous non-increasing function since $w_{3}(\cdot, 0)$ is continuous on $\left[0, T_{0}\right]$. Furthermore, Lemma 5.3 says that $\left|A_{b_{3}}\left(T_{0}\right)\right|=$ $\left|\left\{T_{0}\right\}\right|=0$.

On the other hand, let $\nu_{3}<k<k^{\prime}$, relation (3.2) yields

$$
\begin{aligned}
\left(k^{\prime}-k\right)\left|A_{k^{\prime}}\left(T_{0}\right)\right|^{\frac{1}{3}} & \leq\left\|\left[w_{3}-k\right]^{+}\right\|_{L^{3}\left(0, T_{0}\right)} \\
& \leq c_{3}\left\|\left[w_{3}-k\right]^{+}\right\|_{V_{2}^{1,0}\left(Q_{T_{0}}\right)} .
\end{aligned}
$$

Using the local Lipschitz property (2.6) of function $r$, we have $r\left(v_{1}, v_{2}, v_{3}\right) \leq$ $L v_{3}=L\left(b_{3}-w_{3}\right)$ on the boundary and relation (4.5) yields for $\left.\tau \in\right] 0, T_{0}$ ]

$$
\begin{aligned}
& \frac{1}{2}\left\|\left[w_{3}-k\right]^{+}(\tau)\right\|_{L^{2}(\Omega)}^{2}+\left\|\partial\left[w_{3}-k\right]^{+}\right\|_{L^{2}\left(Q_{\tau}\right)}^{2} \\
& \quad \leq-\int_{0}^{T_{0}} r\left(v_{1}, v_{2}, v_{3}\right)\left(a_{3}-b_{3}+w_{3}-\frac{1}{2}\left[w_{3}-k\right]^{+}\right)\left[w_{3}-k\right]^{+}(t, 0) d t .
\end{aligned}
$$

Using the same technique we have introduced in Lemma 4.1, there exists a constant $C$ such that 


$$
\begin{aligned}
\left\|\left[w_{3}-k\right]^{+}\right\|_{V_{2}^{1,0}\left(Q_{T_{0}}\right)}^{2} \leq & C\left\|a_{3}-b_{3}+w_{3}-\frac{1}{2}\left[w_{3}-k\right]^{+}\right\|_{L^{\infty}\left(0, T_{0}\right)} \\
& \times \int_{A_{k}\left(T_{0}\right)} L\left|b_{3}-w_{3}\right|\left[w_{3}-k\right]^{+} d t \\
\leq & C M L \int_{A_{k}\left(T_{0}\right)}\left|b_{3}-w_{3}\right|\left[w_{3}-k\right]^{+} d t \\
\leq & C M L\left(b_{3}-k\right)^{2}\left|A_{k}\left(T_{0}\right)\right|
\end{aligned}
$$

where the constants $C, M$ and $L$ do not depend on $k$. We obtain then

$$
\left|A_{k^{\prime}}\left(T_{0}\right)\right| \leq\left(C^{\prime} \frac{b_{3}-k}{k^{\prime}-k}\right)^{3}\left|A_{k}\left(T_{0}\right)\right|^{3 / 2}
$$

For every $\theta \in] 0,1\left[\right.$, we set $k_{\theta}=\theta \nu_{3}+(1-\theta) b_{3}$, we have for $k, k^{\prime} \geq k_{\theta}$

$$
\left|A_{k^{\prime}}\left(T_{0}\right)\right| \leq\left(C^{\prime} \frac{b_{3}-k_{\theta}}{k^{\prime}-k}\right)^{3}\left|A_{k}\left(T_{0}\right)\right|^{3 / 2} .
$$

From Lemma 4.3, we know that there exists $d_{\theta}$ such that

$$
\left|A_{k_{\theta}+d_{\theta}}\left(T_{0}\right)\right|=0
$$

with

$$
d_{\theta}=C^{\prime \prime}\left(b_{3}-k_{\theta}\right)\left|A_{k_{\theta}}\left(T_{0}\right)\right|^{1 / 6}
$$

where $C^{\prime \prime}$ does not depend on $\theta$. We get then the estimate

$$
\gamma_{3}\left(T_{0}\right) \leq \theta \nu_{3}+(1-\theta) b_{3}+\theta C^{\prime \prime}\left(b_{3}-\nu_{3}\right)\left|A_{k_{\theta}}\left(T_{0}\right)\right|^{1 / 6} .
$$

Since the limit of $\left|A_{k}\left(T_{0}\right)\right|$ is 0 at point $b_{3}$, we can choose $\theta_{0} \neq 0$ such that

$$
C^{\prime \prime}\left|A_{k_{\theta_{0}}}\left(T_{0}\right)\right|^{1 / 6} \leq \frac{1}{2}
$$

hence we get $\gamma_{3}\left(T_{0}\right) \leq b_{3}-\left(\theta_{0} / 2\right)\left(b_{3}-\nu_{3}\right)<b_{3}$. We have then $w_{3}\left(T_{0}, 0\right)<b_{3}$ and the lemma is proved.

Theorem 5.2 (Global existence and uniqueness). Assume $\nu_{2}<b_{2}$ and $\nu_{3}<b_{3}$, then there exists a unique solution $\left(w_{i}\right)_{i=1}^{3}$ in $W_{2}^{1,2}\left(Q_{T}\right) \cap$ $L^{\infty}\left(Q_{T}\right)$ for problem $\mathcal{P}_{\sqsupseteq}$ with $w_{1} \leq b_{1}, w_{2}<b_{2}, w_{3}<b_{3}$.

Proof. Assuming $T_{0}<T$, Lemmas 5.3 and 5.4 give a contradiction, hence $T_{0}=T$. We obtain the existence of an extension. The local uniqueness and the fact that $w_{i} \in C^{0}\left([0, T] ; L^{2}(\Omega)\right)$ yield the uniqueness of the extended solution. 
Remark 5.3. Theorem 5.2 holds if we reduce the assumption $w_{i}^{0} \in H^{1}(\Omega) \cap$ $L^{\infty}(\Omega)$ to $w_{i}^{0} \in L^{2}(\Omega) \cap L^{\infty}(\Omega)$. Indeed, Theorem 5.1 gives local existence and uniqueness of the solution $\left(w_{1}, w_{2}, w_{3}\right)$ on $\left[0, \delta_{0}\right]$ for a $\delta_{0}>$ 0 . Remark 5.1 says that we have $w_{i} \in L^{2}\left(\left[\delta_{0} / 2, \delta_{0}\right], H^{2}(\Omega)\right)$ and $\partial_{t} w_{i} \in$ $L^{2}\left(\left[\delta_{0} / 2, \delta_{0}\right], L^{2}(\Omega)\right)$. In particular $w_{i}\left(\cdot, \delta_{0} / 2\right) \in H^{1}(\Omega)$. We can then reproduce the proofs in Section 5 after a time translation of $\delta_{0} / 2$ and use Theorem 5.2.

Remark 5.4. Since $\alpha \in H^{1}(0, T)$, the functions $u_{i}=b_{i}-w_{i}(t, y+\alpha(t))$ are bounded and differentiable on every compact domain $K$ of $[\alpha(t), \infty$ [ with $u_{i} \in L^{2}\left(0, T ; H^{1}(K)\right)$. Furthermore, since $\alpha^{\prime}$ is bounded, the functions

$$
\frac{\partial u_{i}}{\partial t}=-\frac{\partial w_{i}}{\partial t}-\alpha^{\prime} \frac{\partial w_{i}}{\partial y}, i=1,2,3
$$

belong to $L^{2}\left(0, T ; H^{-1}(] \alpha(t), \infty[)\right)$. We obtain then a unique solution for the problem $\left(\mathcal{P}_{\sqcap}\right)$ in the weak sense

$$
\int_{\alpha(t)}^{\infty} \partial_{t} u_{i} \phi d x+D \int_{\alpha(t)}^{\infty} \partial_{x} u_{i} \partial_{x} \phi=\alpha^{\prime}\left[a_{i}-b_{i}+u_{i}(t, 0)\right] \phi(0) \quad \forall \phi \in \mathcal{D}(\mathcal{K}) .
$$

Remark 5.5. The bound of functions $u_{1}, u_{2}, u_{3}$ implies that functions $c_{i}$ are bounded and $c_{i} \in L^{2}\left(0, T ; H^{1}(K)\right)$ for all compact $K$ of $[\alpha(t), \infty[$. Relation (1.1) says that the functions $c_{3}, c_{4}, c_{6}$ have the same regularity. Let $\phi$ be a real regular function on $K$, the time derivative vector $\frac{\partial c_{i}}{\partial t}, i=1,2,5$ comes from the matrix product of $[D F(c)]^{-1}$ with the vector $\frac{\partial u_{i}}{\partial t}, i=1,2,3$. All the terms of the matrix $\phi[D F(c)]^{-1}$ belong to $L^{2}\left(0, T ; H^{1}(K)\right)$ hence we can give a sense to the dual product $\left\langle\frac{\partial c_{i}}{\partial t}, \phi\right\rangle$. Therefore, the function $c_{i}$ satisfies relations $(H),(O),(F),(S)$ in a weak sense.

Acknowledgments. This paper has been written at the time of a visit in the Mathematics Department of Lisboa University in Portugal. The author is greatly indebted for Professor J. F. Rodrigues for many stimulating discussions and comments and greatly appreciated the valuable remarks of the referee. The author whish to thank the ESF/FBP programme for its financial support. 


\section{References}

[1] Born, H.H., Prigogine, M., The rate equation for the dissolution of silica in hydrochloric-hydrofluoric acid mixture, Journal de Chimie Physique 76(6) (1979), 538-544.

[2] Bruch, J.C., Papadopoulos, C.A., Sloss, J.M., Parallel computing using in solving wet chemical etching semiconductor fabrication problems, GATUKO Internat. Ser. Math. Sci. Appl. 1 (1993), 281-292.

[3] Brezis, H., Analyse Fonctionnelle, Théorie et Applications, Masson, 1987.

[4] Buck, R.C., Advanced Calculus, 2nd Edition, Internat. Ser. Pure Appl. Math., Mc Graw-Hill, New York, 1965.

[5] Čermák, L., Finite element solution of diffusion problems with a moving boundary in semiconductor device technology, Pitman Res. Notes Math. Ser. 186 (1990), 621-627.

[6] Cuvelier, C., Vuik, C., Numerical solution of an etching problem, J. Comput. Phys. 59 (1985), 247-263.

[7] Fripiat, J.J., Prigogine, M., La réactivité des surfaces de silices, Journal de Chimie Physique 76(1) (1979), 26-34.

[8] Judge, J.S., The etching of thin film dielectric materials, J. Electrochem. Soc. 118 (1971), 19-36.

[9] Kuiken, H.K., Mathematical modelling of etching processes, Pitman Res. Notes in Math. Ser. 185 (1990), 89-109.

[10] Ladyshenskaya, O., Solonnikov, V., Uraltseva, N., Linear and Quasi-Linear Equations of Parabolic Type (English translation), Transl. Math. Monograph., Amer. Math. Soc. 23, Providence, R.I., 1968.

[11] Simon, J., Compacts sets in the spaces $L^{p}(0, T ; B)$, Ann. Mat. Pura Appl. 146(4) (1987), 65-96.

[12] Stampacchia, G., Équations Elliptiques du Second Ordre à Coefficients Discontinues, Les Presses Universitaires de Montréal, 1965.

\section{StÉPhane Clain}

Département de Mathématiques Appliquées

Université Blaise Pascal

63177 Aubière Cedex

E-MAIL CLAIN@UCFMA.UNIV-BPCLERMONT.FR 